Arq. Bras. Med. Vet. Zootec., v.69, n.1, p.191-197, 2017

\title{
Zeólitas naturais na dieta de frangos de corte
}

[Natural zeolites in broiler diet]

\author{
A.F. Schneider, F.M. Yuri, D.S. Almeida, J.V.C. Roeder, J.S. Xavier, C.E. Gewehr \\ Universidade do Estado de Santa Catarina - Lages, SC
}

\begin{abstract}
RESUMO
O objetivo deste trabalho foi avaliar o efeito da inclusão de $0,5 \%$ da zeólita natural clinoptilolita na dieta de frangos de corte sobre o consumo de água, ração e características das excretas. Foi conduzido um ensaio em gaiolas metabólicas, com frangos de corte de linhagem comercial, no período de 14 a 23 dias de idade. $\mathrm{O}$ experimento foi constituído de dois grupos de aves, sendo que um recebeu dieta sem inclusão de zeólitas (controle) e outro dieta com inclusão de 0,5\% de zeólita (clinoptilolita) em rações isonutritivas, em um delineamento inteiramente ao acaso, com 10 repetições de 10 aves. Avaliou-se o consumo de água e de ração, nas excretas, $\mathrm{pH}$, teor de nitrogênio total e umidade. A inclusão de $0,5 \%$ de zeólitas naturais na dieta não alterou $(\mathrm{P}>0,05)$ o consumo de água e de ração e o teor de nitrogênio das excretas, entretanto, reduziu $(\mathrm{P}<0,05)$ o $\mathrm{pH}$ e a umidade das excretas. Conclui-se que a inclusão de $0,5 \%$ de zeólitas naturais na dieta de frangos de corte reduz o $\mathrm{pH}$ e a umidade das excretas e pode ser utilizada como aditivo alimentar sem prejudicar o consumo de água e de ração.
\end{abstract}

Palavras-chave: amônia, clinoptilolita, $\mathrm{pH}$ de excretas, umidade das excretas

\begin{abstract}
The aim of this study was to evaluate the addition of $0.5 \%$ natural zeolites (clinoptilolite) to the diet of broilers and their effect on water and feed intake and excreta characteristics. The test was carried out in metabolic cages, with commercial line broilers, in the period from 14 to 23 days old. The experiment was conducted with two groups of broilers: one group received diet without zeolite inclusion (control) and the other received a diet with $0.5 \%$ inclusion of zeolite (clinoptilolite) in balanced diets, in a completely randomized design, with 10 replicates of 10 broilers. Water and feed intake, $\mathrm{pH}$, total nitrogen, and moisture of excreta were evaluated. The inclusion of $0.5 \%$ of natural zeolites in the diet did not change $(P>0.05)$ the water and feed intake and total nitrogen of excreta, nevertheless, it decreased $(P<0.05)$ the $\mathrm{pH}$ and moisture of excreta. We conclude that the addition of $0.5 \%$ natural zeolites to the diet of broilers decreases $\mathrm{pH}$ and moisture of excreta and can be used as feed additive without compromising water and feed intake.
\end{abstract}

Keywords: Ammonia, clinoptilolite,moisture of excreta, $\mathrm{pH}$ of excreta

\section{INTRODUÇÃO}

Zeólitas são grupos de minerais cristalinos, constituídos de estrutura tridimensional de tetraedros interligados, compostos por quatro átomos de oxigênio em torno de um cátion, o que lhes confere características peculiares. Os canais são frequentemente ocupados por moléculas de água e por cátions (Coombs et al., 1997) e em seus microporos podem fluir moléculas que

Recebido em 2 de setembro de 2015

Aceito em 7 de julho de 2016

E-mail: aline_schneider@hotmail.com possuam dimensão inferior a um valor crítico (Luz, 1995).

Dentre as propriedades das zeólitas destacam-se a capacidade de adsorção de íons, de troca catiônica e de absorção de água. As zeólitas também são utilizadas como peneiras moleculares em processos de remoção de impurezas ou separação de moléculas, devido ao seu volume poroso e ao diâmetro dos poros (Aguiar e Novaes, 2002). Em meio a esses 
processos, uma peculiaridade é a alta afinidade e seletividade das zeólitas por íons $\mathrm{NH}_{4}^{+}$(amônio), resultante da capacidade de troca cationnica e da estrutura química desses minerais (Mumpton e Fishman, 1977). A adsorção do amônio pelas zeólitas in vitro foi comprovada por Ly et al. (1996). Esses mesmos autores destacam o efeito benéfico das zeólitas nos processos digestivos por impedir a possível absorção intestinal da amônia, já que esse gás é tóxico e, quando absorvido, passa por reações que envolvem gasto enérgico até sua eliminação. Porém, o mecanismo pelo qual as zeólitas clinoptilolitas agem no nível intestinal ainda precisa ser estudado (Wu et al., 2013).

Visando à redução da poluição ambiental, a produção animal deve atentar para as características das excretas produzidas pelas criações. Diante dessa problemática, buscam-se alternativas, como a utilização de aditivos alimentares, condicionadores de cama e de excretas que possam apresentar efeito benéfico tanto para a indústria avícola quanto para o meio ambiente (Burgos e Burgos, 2006). As propriedades das zeólitas fazem com que elas sejam testadas como aditivos inorgânicos na dieta de diversas espécies animais, buscando melhores resultados de desempenho zootécnico, adsorção a micotoxinas, qualidade de excretas, entre outros. Possíveis melhorias na qualidade das excretas das aves implicam diretamente nas características da cama do aviário, principalmente nos teores de umidade e volatilização da amônia, os quais são nocivos à saúde e acarretam prejuízos sobre o desempenho das aves.

Com base no exposto, o objetivo deste trabalho foi avaliar o efeito da inclusão de $0,5 \%$ da zeólita natural clinoptilolita na dieta de frangos de corte sobre o consumo de água e ração e as características de suas excretas.

\section{MATERIAL E MÉTODOS}

Um experimento foi conduzido no Setor de Avicultura do Centro de Ciências Agroveterinárias da Universidade do Estado de Santa Catarina, localizado nas coordenadas geográficas $27^{\circ} 48^{\prime} 11.9^{\prime \prime S}$ e $50^{\circ} 18^{\prime} 17.9^{\prime \prime} \mathrm{W}$.

Foram utilizados 300 pintos de corte de um dia, de linhagem comercial, os quais foram inicialmente alojados em pinteiro único, recebendo água e ração isonutritiva ad libitum, formuladas de acordo com as recomendações (Tab. 1) de Rostagno et al. (2005). Aos 14 dias de idade, foram selecionadas 200 aves com 506g $\pm 5 \%$ de variação em relação ao peso médio. As aves foram distribuídas aleatoriamente em 20 gaiolas metabólicas $(1,0 \times 0,5 \mathrm{~m})$, dotadas de comedouro tipo calha, dois bebedouros tipo nipple acoplados a um reservatório individual de água e uma bandeja coletora de excretas na parte inferior. $\mathrm{O}$ ambiente foi climatizado de acordo com a temperatura de conforto das aves, conforme a recomendação do manual da linhagem.

O ensaio foi conduzido com um período de adaptação de quatro dias (14 a 17 dias de idade) e um período de avaliação de cinco dias (18 a 23 dias de idade). As aves foram submetidas a duas dietas (tratamentos), sendo uma sem inclusão de zeólitas (controle) e outra com inclusão de $0,5 \%$ de zeólitas naturais. Cada tratamento contou com 10 repetições de 10 aves. A composição nutricional e calculada das rações está apresentada na Tab. 1. Utilizou-se a zeólita natural do grupo das clinoptilolitas composta, em sua maioria, por $\mathrm{SiO}_{2} \mathrm{eAl}_{2} \mathrm{O}_{3}$. O mineral continha granulometria de 0,4 a $1 \mathrm{~mm}, \mathrm{pH} 7,6$, e capacidade de troca catiônica de $1,57 \mathrm{meq} / \mathrm{g}$.

Para avaliar o consumo de água, foi disposto um reservatório de $3 \mathrm{~L}$ sobre cada gaiola, acoplado ao bebedouro nipple, o qual era abastecido diariamente, e, no dia seguinte, a quantidade de água restante era retirada e pesada em balança analítica de precisão $(0,001 \mathrm{~g})$. Dividiu-se o consumo total da gaiola pelo número de aves, obtendo-se o consumo de água expresso em $\mathrm{mL} / \mathrm{ave} / \mathrm{dia}$, sendo o fornecimento ad libitum.

O consumo de ração foi obtido por meio do fornecimento ad libitum de uma quantidade conhecida e, ao final dos quatro dias de coleta, as sobras foram pesadas, obtendo-se o consumo geral da gaiola, o qual foi dividido pelo número de aves e expresso em g/ave/dia. A relação consumo de água/ração foi calculada pela razão entre o consumo de água e o consumo de ração de cada repetição.

Do $18^{\circ}$ ao $22^{\circ}$ dia, as excretas eram coletadas diariamente, acondicionadas em sacos plásticos e congeladas a $-18^{\circ} \mathrm{C}$. Para avaliação de potencial 
hidrogeniônico (pH) e nitrogênio total das excretas, as amostras foram descongeladas em temperatura ambiente por $24 \mathrm{~h}$, com os sacos plásticos ainda fechados e, em seguida, colocadas em recipientes apropriados, onde foram homogeneizadas manualmente. $\mathrm{O} \mathrm{pH}$ foi determinado por meio da metodologia adaptada de Oliveira et al. (2003), utilizando-se $30 \mathrm{~g}$ de excretas, as quais foram maceradas em um béquer contendo $250 \mathrm{~mL}$ de água deionizada. Após, a amostra foi deixada em repouso por cinco minutos, e posteriormente realizou-se leitura em pH-metro digital (Tecnopon MPA 210, versão 7.1). O nitrogênio total das excretas foi determinado pelo método Kjeldhal (Silva e Queiroz, 2004).

Tabela 1. Composição nutricional e calculada das rações experimentais

\begin{tabular}{lccc} 
& \multicolumn{2}{c}{ Ingredientes (\%) } & \multicolumn{2}{c}{ Inicial (8 a 23 dias) } \\
\cline { 2 - 4 }$(1 \mathrm{a} 7$ dias $)$ & - & Controle & Suplementada \\
\hline Milho & 60,3 & 63,8 & 62,7 \\
Farelo de soja & 30,7 & 27,8 & 28,0 \\
Farinha de carne & 7,24 & 6,73 & 6,74 \\
Farinha de ostra & 0,26 & 0,27 & 0,26 \\
Cloreto de sódio & 0,18 & 0,18 & 0,17 \\
Óleo de soja & 0,47 & 0,59 & 0,94 \\
Metionina & 0,18 & 0,12 & 0,12 \\
Lisina & 0,17 & 0,07 & 0,07 \\
Treonina & 0,03 & - & - \\
Zeólitas & - & - & 0,50 \\
Premix mineral e vitamínico ${ }^{1}$ & 0,44 & 0,44 & 0,44 \\
\hline & & Composição calculada & 3.000 \\
\hline Energia metabolizável - kcal/kg & 2.950 & 3.000 & 20,8 \\
Proteína - \% & 22,0 & 20,8 & 0,88 \\
Cálcio - \% & 0,94 & 0,88 & 0,44 \\
Fósforo disp. - \% & 0,47 & 0,44 & 1,15 \\
Lisina disp. - \% & 1,33 & 1,15 & 0,44 \\
Metionina disp. - \% & 0,52 & 0,44 & \\
\hline
\end{tabular}

${ }^{\mathrm{I}}$ Níveis por kg do produto: vitamina B12 3.000,00mcg, vitamina B6 622,00mg, ácido pantotênico 2.934,9mg, niacina $7.500,00 \mathrm{mg}$, biotina $19,00 \mathrm{mg}$, vitamina B2 $1.125,00 \mathrm{mg}$, manganês $16.800,0 \mathrm{mg}$, zinco $13.000,00 \mathrm{mg}$, ferro $12.600,00 \mathrm{mg}$, iodo $250,00 \mathrm{mg}$, cobre $2.100,00 \mathrm{mg}$, selênio $75,00 \mathrm{mg}$, vitamina A $2.640 .000,00 \mathrm{UI} / \mathrm{kg}$, vitamina D3 $638.000,00 \mathrm{UI} / \mathrm{kg}$, vitamina E 3.650,00UI/kg, vitamina K3 450,00mg, vitamina B1 502,00mg, ácido fólico 189,00mg, colistina $2.500,00 \mathrm{mg}$, BHT $0,80 \mathrm{~g}$, enramicina $2.500,00 \mathrm{mg}$, semduramicina $6.250,00 \mathrm{mg}$, colina $86,67 \mathrm{~g}$.

Para avaliação de umidade, procedeu-se à coleta das excretas no $23^{\circ}$ dia, sendo as bandejas limpas, colocadas ordenadamente nas gaiolas e retiradas após duas horas. As excretas foram acondicionadas em sacos plásticos identificados e, em seguida, congeladas a $-18^{\circ} \mathrm{C}$. Adotou-se esse procedimento com o objetivo de minimizar interferências de variáveis ambientais, com possível evaporação de água das excretas ou absorção de umidade do meio externo. A umidade foi aferida por meio de pesagem inicial das excretas, e a posterior secagem em estufa com ventilação forçada a $105^{\circ} \mathrm{C}$ por $24 \mathrm{~h}$ (Silva e Queiroz, 2004).

As análises estatísticas foram realizadas utilizando-se o delineamento inteiramente ao acaso, sendo a gaiola com 10 aves considerada como unidade experimental, adotando-se o modelo de análise de variância e o teste $\mathrm{t}$ de Student $(5 \%)$, analisados pelo sistema de análise estatística e planejamento de sistemas - Sisvar (Ferreira, 2011).

O projeto foi submetido ao Comitê de Ética em Experimentação Animal - Cetea, da Universidade do Estado de Santa Catarina, e aprovado sob o número de protocolo 1.25.12.

\section{RESULTADOS E DISCUSSÃO}

$\mathrm{O}$ consumo de água não foi alterado $(\mathrm{P}>0,05)$ com a inclusão de $0,5 \%$ de zeólitas na dieta das aves (Tab. 2). Aditivos ricos em minerais podem 
aumentar a osmolaridade e, consequentemente, o consumo de água (Russel e Chow, 1993), e quanto maior o consumo hídrico, maior será a umidade da cama do aviário (Borges et al., 2002), o que torna essa avaliação importante.

A literatura reporta poucos trabalhos que avaliam o efeito da inclusão de zeólitas nas dietas sobre o consumo de água. Em um trabalho realizado com galinhas Leghorn de 48 dias de idade, durante um período de duas semanas, com a inclusão de clinoptilolita e mordenita nos níveis 3, 5 e $10 \%$ na dieta, observou-se menor consumo de água e ração sem alterar o ganho de peso em relação às aves que receberam dieta sem a suplementação desses minerais (Onagi, 1966). A ingestão de água depende da idade das aves e do nível de sais na dieta (Borges et al., 2002), e o consumo de água afeta diretamente a ingestão de alimentos. Assim, restrições hídricas resultarão em efeito negativo sobre o desempenho da ave.

Tabela 2. Consumo diário de água e ração, características das excretas de frangos de corte alimentados com dietas sem e com a suplementação com $0,5 \%$ de zeólitas

\begin{tabular}{lcccc}
\hline & Controle & Suplementada & CV (\%) & P \\
\cline { 2 - 5 } & Dados zootécnicos & & & \\
\hline Consumo de água - mL/ave/dia & 194,9 & 197,6 & 7,26 & 0,653 \\
Consumo de ração - g/ave/dia & 111,5 & 107,9 & 4,45 & 0,104 \\
Relação consumo água/ração & 1,75 & 1,83 & 9,00 & 0,236 \\
\hline \multicolumn{5}{c}{ Características das excretas } \\
Nitrogênio total - \% & $5,88 \mathrm{a}$ & $5,81 \mathrm{~b}$ & 0,98 & 0,018 \\
Umidade - \% & 1,49 & 1,55 & 9,99 & 0,415 \\
\hline
\end{tabular}

Letras desiguais na linha diferem pelo teste $\mathrm{T}(\mathrm{P}<0,05)$

CV (\%): coeficiente de variação.

P: probabilidade.

Não foram observadas diferenças $(\mathrm{P}>0,05)$ sobre o consumo de ração (Tab. 2), o que corrobora os resultados obtidos por outros autores (Karamanlis et al., 2008; Çabuk et al., 2004), que testaram dosagens superiores à avaliada no presente trabalho. A inclusão de $2 \%$ de zeólitas na dieta de frangos de corte não alterou $o$ consumo de ração de aves alimentadas até os 28 dias de idade (Karamanlis et al., 2008), tampouco quando foram incluídas na dieta de frangos de corte nas doses zero, 1,5 e 2,5\% (Çabuk et al., 2004). A inclusão de zeólitas na dieta das aves pode resultar em diversos efeitos favoráveis, como proteção contra micotoxinas, retenção da umidade da ração e, consequentemente, menor predisposição ao desenvolvimento de fungos, além de proporcionar menor compactação das partículas da ração (Herzig et al., 2008).

A relação entre o consumo de água e de ração (Tab. 2) manteve-se sem alterações $(\mathrm{P}>0,05)$. Esse resultado reflete e corrobora os resultados obtidos nas duas variáveis descritas anteriormente, o que atesta que a inclusão de zeólitas na dieta não altera o consumo de água ou de ração das aves. Em frangos de corte, os consumos de água e de ração são altamente correlacionados, e, por conseguinte, a restrição de um altera diretamente a do outro. O consumo de água apresenta forte influência sobre a restrição alimentar (Soares et al., 2007).

Observou-se redução $(\mathrm{P}<0,05)$ no $\mathrm{pH}$ das excretas com a inclusão de zeólitas na dieta (Tab. 2). $\mathrm{O} \mathrm{pH}$ das excretas tem relação direta sobre o $\mathrm{pH}$ da cama de aviário, sendo determinante no processo de emissão da amônia nos galpões onde as aves são criadas. A volatilização da amônia pode ser entendida como o transporte da fase aquosa ou sólida para a fase gasosa no ar. $\mathrm{O} \mathrm{pH}$ indica sua concentração na fase aquosa ou gasosa, determinando sua liberação. Por sua vez, a acidificação do $\mathrm{pH}$ das excretas contribui para a redução do $\mathrm{pH}$ da cama, o que é um fator importante, já que a liberação de amônia é minimizada em pH abaixo de 7,0 (Carvalho et al., 2011). As características das excretas das aves influenciam diretamente a qualidade da cama do aviário e, por isso, os possíveis efeitos de um aditivo alimentar sobre as excretas devem ser avaliados. 
O teor de nitrogênio das excretas (Tab. 2) não foi alterado $(\mathrm{P}>0,05)$ com a inclusão de zeólitas na dieta. $\mathrm{O}$ nitrogênio é um fator importante no processo de volatilização de amônia, já que age como substrato para a produção desse gás. Alguns estudos demonstram que, sempre que houver maiores níveis de nitrogênio disponíveis, haverá maior teor de volatilização de amônia (Egute et al., 2010). Diferentemente dos resultados encontrados neste estudo, Machacek et al. (2010) verificaram maior utilização metabólica de nitrogênio em poedeiras recebendo dieta com inclusão de $2 \%$ de zeólitas, porém os autores utilizaram dose quatro vezes superior ao presente trabalho.

A capacidade de troca catiônica e a adsorção de íons são propriedades das zeólitas que podem ser mais exploradas para se fazer um melhor aproveitamento do nitrogênio na nutrição animal (Mumpton e Fishman, 1977). A utilização da zeólita clinoptilolita com inclusão de 1,2\% na dieta de bovinos confinados não afetou o balanço de nitrogênio e a volatilização, e também não se observaram diferenças no nitrogênio presente nas fezes ou perdido. Destaca-se que, em outras espécies animais, as zeólitas são efetivas na adsorção de nitrogênio e que as divergências nos resultados dos trabalhos podem estar relacionadas a variações nos minerais utilizados ou nas metodologias empregadas para quantificação de nitrogênio (Sherwood et al., 2006).

A umidade das excretas (Tab. 2) das aves foi reduzida $(\mathrm{P}<0,05)$ com a inclusão de zeólitas, evidenciando o efeito higroscópico desses minerais. Essa resposta era esperada, já que grande parte dos canais e das cavidades das zeólitas é preenchida por moléculas de água (Mohebodini, 2008). E importante avaliar a umidade das excretas das aves ao se incluir minerais na dieta, uma vez que eletrólitos podem ser responsáveis pelo aumento na umidade delas (Vieites et al., 2005). A inclusão de 3\% de zeólitas na dieta de frangos de corte criados até 40 dias de idade provocou redução da umidade fecal de 79,14 para 73,01\%, em comparação com as aves que receberam dieta sem zeólitas (Safaei katouli et al., 2010). A inclusão de 0,5;0,75 e $1,0 \%$ de zeólitas na dieta de cães adultos indicou que doses acima de $0,75 \%$ proporcionaram maior teor de matéria seca e melhor classificação do escore fecal, sendo o resultado atribuído à capacidade higroscópica das zeólitas (Maia et al., 2010). Miles e Henry (2007) citam que a umidade das excretas diminui linearmente de acordo com o aumento de aluminossilicato na dieta, o que é coerente e corrobora os resultados dos trabalhos supracitados. A maioria das zeólitas retém água por hidratação dos cátions que estão compensando cargas superficiais ou por balanço osmótico (Castaing, 1998).

O menor teor de umidade encontrado nas excretas de aves que consumiram dieta com zeólitas é um resultado interessante, pois a alta umidade implica piora da qualidade da cama do aviário (Borges et al., 2002). Isso ficou evidente em um experimento que resultou em menor umidade da cama das aves com inclusão das doses 1,2 e $3 \%$ de zeólitas na dieta de frangos de corte, sendo esse resultado atribuído à absorção da umidade das excretas (Nikolakakis et al., 2013). Nesse aspecto, a utilização de aditivos alimentares pode contribuir para a mitigação do impacto ambiental da produção de frangos de corte por efeito direto nas excretas das aves, sendo as zeólitas um exemplo para essa condição (Oviedo-Rondón, 2008).

Observa-se que os resultados encontrados na literatura divergem em função da dose de inclusão, da espécie animal e das características das zeólitas utilizadas nos ensaios. É importante destacar que, neste trabalho, incluiu-se somente $0,5 \%$ na dieta por ser considerada uma dose viável para ser recomendada em criações comerciais. Não seria interessante uma inclusão superior a $0,5 \%$, pois implicaria aumento no custo da dieta decorrente não somente do custo das zeólitas, mas também do aumento da concentração dos ingredientes principais na dieta, com, por exemplo, maior inclusão de óleo para manter o nível de energia metabolizável requerido, o qual possui elevado valor comercial.

\section{CONCLUSÃO}

Conclui-se que a inclusão de $0,5 \%$ da zeólita natural clinoptilolita na dieta de frangos de corte reduz o $\mathrm{pH}$ e a umidade das excretas e pode ser utilizada como aditivo alimentar sem afetar o consumo de água e de ração. 


\section{REFERÊNCIAS}

AGUIAR, M.R.M.P.; NOVAES, A.C. Remoção de metais pesados de efluentes industriais por aluminossilicatos. Quím. Nova, v.25, p.11451154, 2002.

BORGES, S.A.; SALVADOR, D.; SILVA, A.V.F.; BUENO, F.L. Consumo de água em frangos de corte suplementados com bicarbonato de sódio ou cloreto de potássio na ração. Ciênc. Cult., n.31, p.89-96, 2002.

BURGOS, S.; BURGOS, S.A. Environmental approaches to poultry feed formulation and management. Poult. Sci., v.5, p.900-904, 2006.

CARVALHO, T.M.R.; MOURA, D.J.; SOUZA, Z.M. et al. Qualidade da cama e do ar em diferentes condições de alojamento de frangos de corte. Pesqui. Agropecu. Bras., v.46, p.351-361, 2011.

CASTAING, J. Uso de las arcillas en alimentación animal. In: CURSO DE ESPECIALIZACIÓN VANCES EN NUTRICIÓN Y ALIMENTACIÓN ANIMAL, 14., 1998, Fira de Barcelona. Anal... Fira de Barcelona: [DIALNET], 1998. p.141-158. Disponible el: $<$ http://www.etsia.upm.es/fedna/capitulos/98CA PVIII.pdf $>$. Consultado el: 15 dic. 2012.

COOMBS, D.S.; ALBERTI, A.; ARMBRUSTER, T. et al. Recommended nomenclature for zeolite minerals: report of the subcommittee on zeolites of the international mineralogical association, commission on new minerals and mineral names. Can. Mineral., v.35, p.1571-1606, 1997.

ÇABUK, M.; ALÇIÇEK, A.; BOZKURT, M.; AKKAN, S. Effect of yucca schidigera and natural zeolite on broiler performance. Poult. Sci., v.10, p.651-654, 2004.

EGUTE, N.S.; ABRÃO, A.; CARVALHO, F.M.S. Estudo do processo de geração de amônia a partir de resíduos avícolas visando a produção de hidrogênio. Rev. Bras. Pesqui. Desenvol., v.12, p.1-6, 2010.

FERREIRA, D.F. Sisvar: a computer statistical analysis system. Ciênc. Agrotecnol. v.35, p.1039-1042, 2011.
HERZIG, I.; STRAKOVA, E.; SUCHY, P. Long-term application of clinoptilolite via the feed of layers and its impact on the chemical composition of long bones of pelvic limb (femur and tibiotarsus) and eggshell. Vet. Med., v.53, p.550-554, 2008.

KARAMANLIS, X.; FORTOMARIS, P.; ARSENOS, G. et al. A. The effect of natural zeolite (clinoptilolite) on the performance of broiler chickens and the quality of their litter. Asian-Aust. J. Anim. Sci., v.21, p.1642-1650, 2008.

LUZ, A.B. Zeólitas: propriedades e usos industriais. Rio de Janeiro: CETEM/CNPQ, 1995. 35p. (Série Tecnologia Mineral, 68).

LY, J.; CARÓN, M.; CASTRO, M.; LON-WO, E. Una nota sobre la adsorcion in vitro de amoniaco en zeólitas naturales cubanas. Rev. Comput. Prod. Porcina, v.3, p.62-68, 1996.

MACHACEK, M.; VECEREK, V.; MAS, N. et al. Effect of the feed additive clinoptilolite (zeofeed) on nutrient metabolism and production performance of laying hens. Acta Vet., v.79, p.29-34, 2010.

MAIA, G.V.C.; SAAD, F.M.O.B.; ROQUE, N.C. et al. Zeólitas e yucca schidigera em rações para cães: palatabilidade, digestibilidade e redução de odores fecais. Rev. Bras. Zootec., v.39, p.2442-2446, 2010.

MILES, R.D.; HENRY, P.R. Safety of improved Milbond-TX ${ }^{\circledR}$ when fed in broiler diets at greater than recommended levels. Anim. Feed Sci. Technol., v.138, p.309-317, 2007.

MOHEBODINI, H. Zeolite as a feed additive in broiler chickens diets. IRAN INTERNATIONAL ZEOLITE CONFERENCE, 2008, [s.1.]. Proceedings... [s.1.]: [s.n.], 2008. Available in: <http://www.civilica.com/EnPaperZEOLITE01-ZEOLITE01 047.html>. Accessed in: 20 May 2010.

MUMPTON, F.A.; FISHMAN, P.H. The application of natural zeolites in animal science and aquaculture. J. Anim. Sci., v.45, p.11881203, 1977.

NIKOLAKAKIS, I.; DOTAS, V.; KARGOPOULOS, A. et al. Effect of natural zeolite (clinoptilolite) on the performance and litter quality of broiler chickens. Turk. J. Vet. Anim. Sci., v.37, p.682686, 2013. 
OLIVEIRA, M.C.; ALMEIDA, C.V.; ANDRADE, D.O.; RODRIGUES, S.M.M. Teor de matéria seca, $\mathrm{pH}$ e amônia volatilizada da cama de frango tratada ou não com diferentes aditivos. Rev. Bras. Zootec., v.32, p.951-954, 2003.

ONAGI, T. Treating experiment of chicken dropping with zeolite tuff powder. Experimental use of zeolite tuffs as dietary supplements for chicken. Repub. Yamagata Stock Raising Inst., p.7-18, 1966.

OVIEDO-RONDÓN, E.O. Tecnologias para mitigar o impacto ambiental da produção de frangos de corte. Rev. Bras. Zootec., v.37, p.239252, 2008.

ROSTAGNO, H.S.; ALBINO, L.F.T.; DONZELE, J.L. et al. Tabelas brasileiras para aves e suínos: composição de alimentos e exigências nutricionais. Viçosa: UFV, 2005. $186 \mathrm{p}$.

RUSSELL, J.B.; CHOW, J.M. Another theory for the action of ruminal buffer salts: decreased starch fermentation and propionate production. $J$. Anim. Sci., v.76, p.826-830, 1993.

SAFAEI KATOULI, M.; BOLDAJI, F.; DASTAR, B.; HASSANI, S. Effect of different levels of kaolin, bentonite and zeolite on broilers performance. J. Biol. Sci., v.10, p.58-62, 2010.
SHERWOOD, D.M.; ERICKSON, G.E.; KLOPFENSTEIN, T.J. Nitrogen mass balance and cattle performance of steers fed clinoptilolite zeolite clay. Nebr. Beef Cattle Rep., v.1, p.90-91, 2006.

SILVA, D.J.; QUEIROZ, A.C. Análise de alimentos: métodos químicos e biológicos. Viçosa: UFV, 2004. 235p.

SOARES, L.F.; RIBEIRO, A.M.L.; PENZ JÚNIOR, A.M.; GHIOTTI, A. Influência da restrição de água e ração durante a fase préinicial no desempenho de frangos de corte até os 42 dias de idade. Rev. Bras. Zootec., v.36, p.1579-1589, 2007.

VIEITES, F.M.; MORAES, G.H.K.; ALBINO, L.F.T. et al. Balanço eletrolítico e níveis de proteína bruta sobre o desempenho, o rendimento de carcaça e a umidade da cama de frangos de corte de 1 a 42 dias de idade. Rev. Bras. Zootec., v.34, p.1990-1999, 2005.

WU, Q.J.; ZHOU, Y.M.; WU,Y.N.; WANG, T. Intestinal development and function of broiler chickens on diets supplemented with clinoptilolite. Asian-Aust. J. Anim. Sci., v.26, p.987-994, 2013. 